\title{
Successful Resection of Intracardiac Invasive Thymoma with Right Ventricular Inflow Tract Occlusion
}

\author{
Shin-ichiro Hayashi, Mitsuaki Isobe, Motonori Hayashi, Hiroshi Imamura, Tomonobu KoIzumi, \\ Tsuneo NAKAJIMA* and Jun AMANO*
}

\begin{abstract}
A 72-year-old man presenting with the superior vena cava syndrome and intracardiac mass was admitted to our hospital. The mass was resected and confirmed to be invasive thymoma. Three years later, he was re-admitted with recurrence into the intracardiac space without any changes in the anterior mediastinum mass. The mass occupied the right atrial cavity and protruded into the right ventricle, causing right ventricular inflow tract obstruction. He underwent re-operation and irradiation. His postoperative course was uneventful, and he has remained alive. Invasive thymoma with intracardiac extension is extremely rare. (Internal Medicine 39: 139-142, 2000)
\end{abstract}

Key words: mediastinal tumor, intracardiac extension, tricuspid valve obstruction, echocardiography

\section{Introduction}

Thymoma, the most common tumor of the anterior-superior mediastinum (1), is malignant in about one-third of the cases (2). Local invasion occurs most commonly into the pleura $(30 \%)$, pericardium (25\%), lungs $(8 \%)$, and recurrent laryngeal nerve (4\%), but invasion into the intracardiac and great vessels is extremely rare (3). Only a few cases of intracardiac extension of thymoma have been reported in the English literature (3-9). We report a case of invasive thymoma which extended into the right atrium and right ventricle, causing right ventricular inflow tract obstruction.

\section{Case Report}

A 72-year-old man presented with progressive dyspnea and edema of the face and upper extremities in September 1994. Examination revealed engorged neck veins and marked edema in the upper trunk and extremities. There were no signs of neurological abnormalities. Chest radiograph taken on admission showed a wide upper mediastinum. A contrast enhanced computed tomographic (CT) scan of the thorax revealed a solid anterior-superior mediastinal mass that filled the lumen of the superior vena cava (SVC), right atrium and right ventricle (Fig. 1A). He underwent surgery on the basis of a presumptive diagnosis of an anterior mediastinal tumor with intracardiac extension. A median sternotomy was performed, which revealed a firm and nodular tumor infiltrating the left brachiocephalic vein and SVC.

Cardiopulmonary bypass was established by cannulating the right femoral artery and femoral vein. Opening the right atrium revealed a large tumor extending to the right ventricle across the tricuspid orifice. It was attached to the atrial septum and obstructed the tricuspid valve. This area was resected at a sharp angle. The left brachiocephalic vein was incised, and the tumor was severed at this point. The surgeon's fingers were then inserted from the right atrium and from within the left brachiocephalic vein. The tumors in the SVC and the left brachiocephalic vein were then removed by pushing them with the fingers into the right atrium. We concluded, from the extent of the tumors, that radical surgery would be impossible. We therefore focused on saving the life of the patient by resecting the intracardiac tumor, and did not resect the anterior mediastinal tumor. Postoperative echocardiography showed that the right atrium and right ventricle were almost tumor-free. A contrast enhanced CT scan of the thorax revealed the tumor in the SVC had been completely removed. Histology of the excised intracardiac tumor showed a mixed type thymoma with predominantly epithelial components (Fig. 2). The epithelial cells consisted of polygonal (oval) and spindle cells (10). The vessels were surrounded by an empty space containing a few lymphocytes. The presence of such perivascular spaces in a mediastinal tumor is characteristic of thymoma. Mitotic activity was low. Immunohistochemically, the tumor cells were positive for keratin, but negative for vimentin and desmin $(10,11)$. Lymphocytes showed diffuse positive reactions for CD45RO. The

From the First Department of Internal Medicine and *the Second Department of Surgery, Shinshu University School of Medicine, Matsumoto

Received for publication May 14, 1999; Accepted for publication August 31, 1999

Reprint requests should be addressed to Dr. Mitsuaki Isobe, the First Department of Internal Medicine, Shinshu University School of Medicine, 3-1-1, Asahi, Matsumoto, Nagano 390-8621 
A: First admission
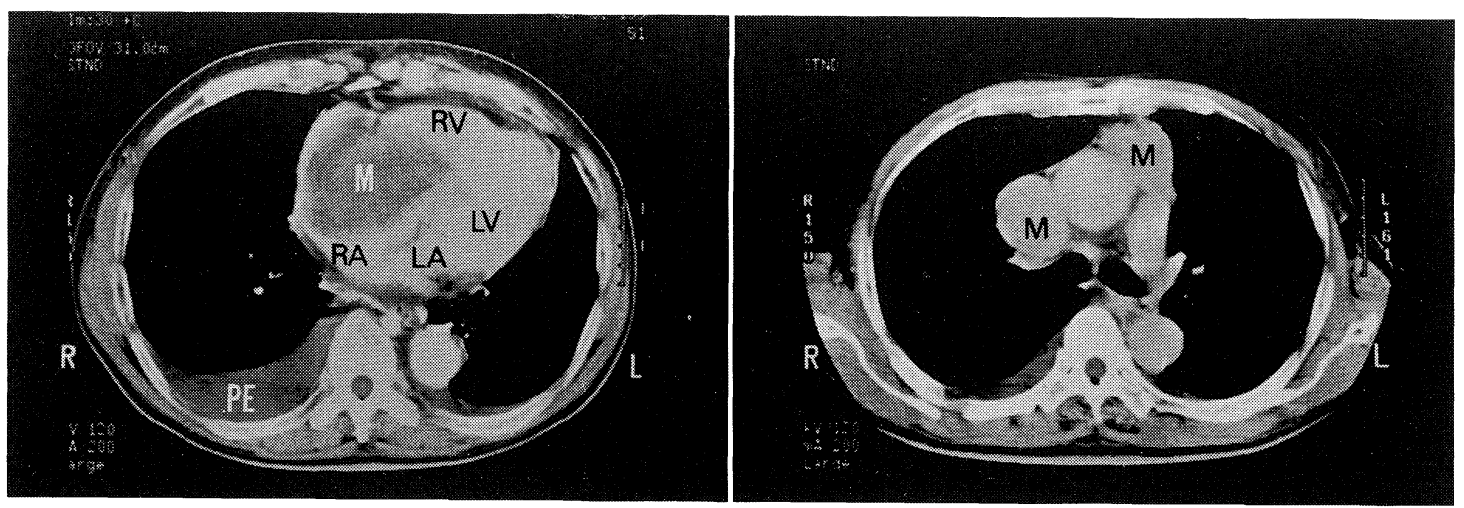

B: Second admission
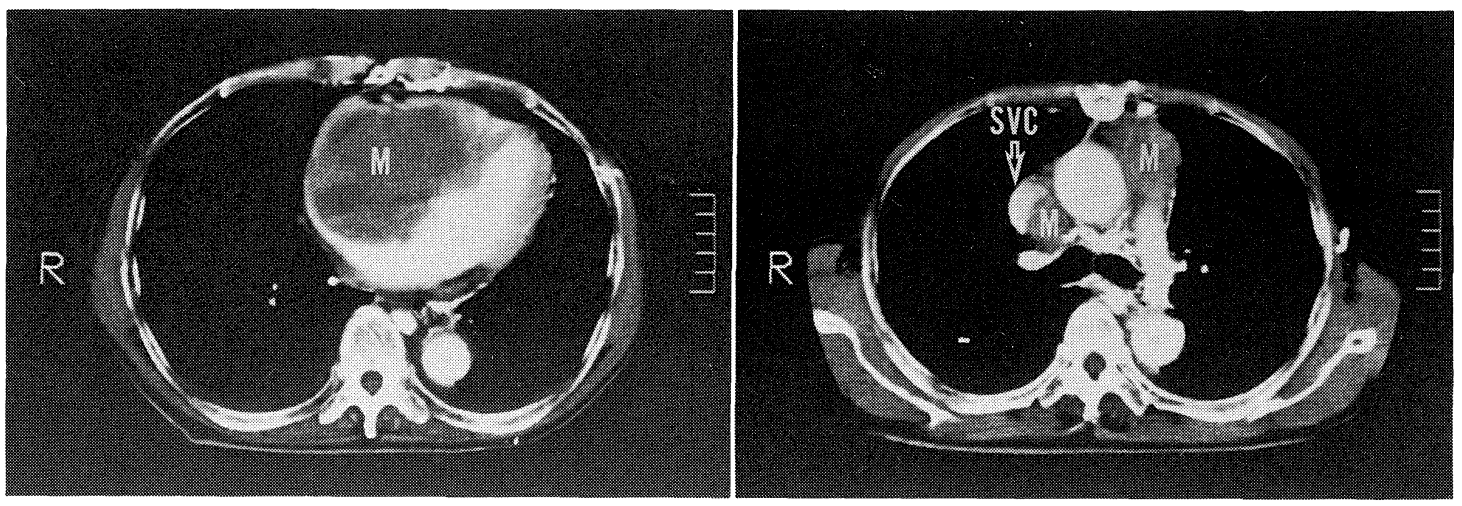

Figure 1. Contrast enhanced CT of the thorax showing the anterior mediastinum mass invading the superior vena cava and the intra cardiac mass in the right atrium and right ventricle at the first admission (A). Contrast enhanced CT of the thorax at the second admission three years later (B). (M: mass, RA: right atrium, RV: right ventricle, LA: left atrium, LV: left ventricle, PE: pleural effusion).

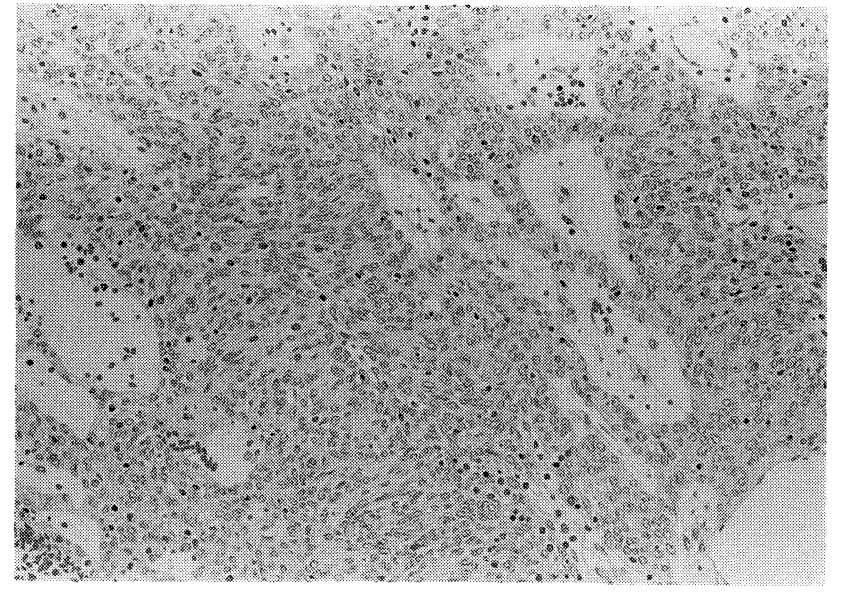

Figure 2. Histology of the excised intracardiac mass revealing mixed-type thymoma with epithelial cells and lymphocytes. Diffuse growth of polygonal and short spindle cells is associated with abundant sinusoidal vasculatures. Perivascular spaces containing a few lymphocytes can been seen (HE stain, $\times 125)$. patient refused postoperative therapy and was discharged in October 1994.

He continued follow-up as an out-patient until October 1995, when he was lost to follow-up. However, on August 7, 1997, he was re-admitted to our hospital with recurrence of rightsided heart failure. Examination revealed SVC syndrome. A grade 3 diastolic rumbling murmur with inspiratory accentuation was audible. CT scan of the thorax showed re-growth of the intracardiac mass, but the size of the mediastinal tumor was not changed. Neither lung nor distant metastasis was detected (Fig. 1B). Echocardiography revealed a large mass measuring $11 \times 6 \mathrm{~cm}$ occupying most of the right atrial cavity. It was attached to the atrial septum and protruded into the right ventricle, causing right ventricular inflow tract obstruction (Fig. 3A). Re-operation involved a right intercostal thoracotomy, and under cardiopulmonary bypass with the same method of first operation, the intracardiac mass was resected. Postoperative echocardiography showed that the right atrium and right ventricle were almost tumor-free with the remainder of the tumor at the base of the atrial septum, as indicated by an arrow in Fig. $3 \mathrm{~B}$. Histology of the resected tumor was compatible with thy- 

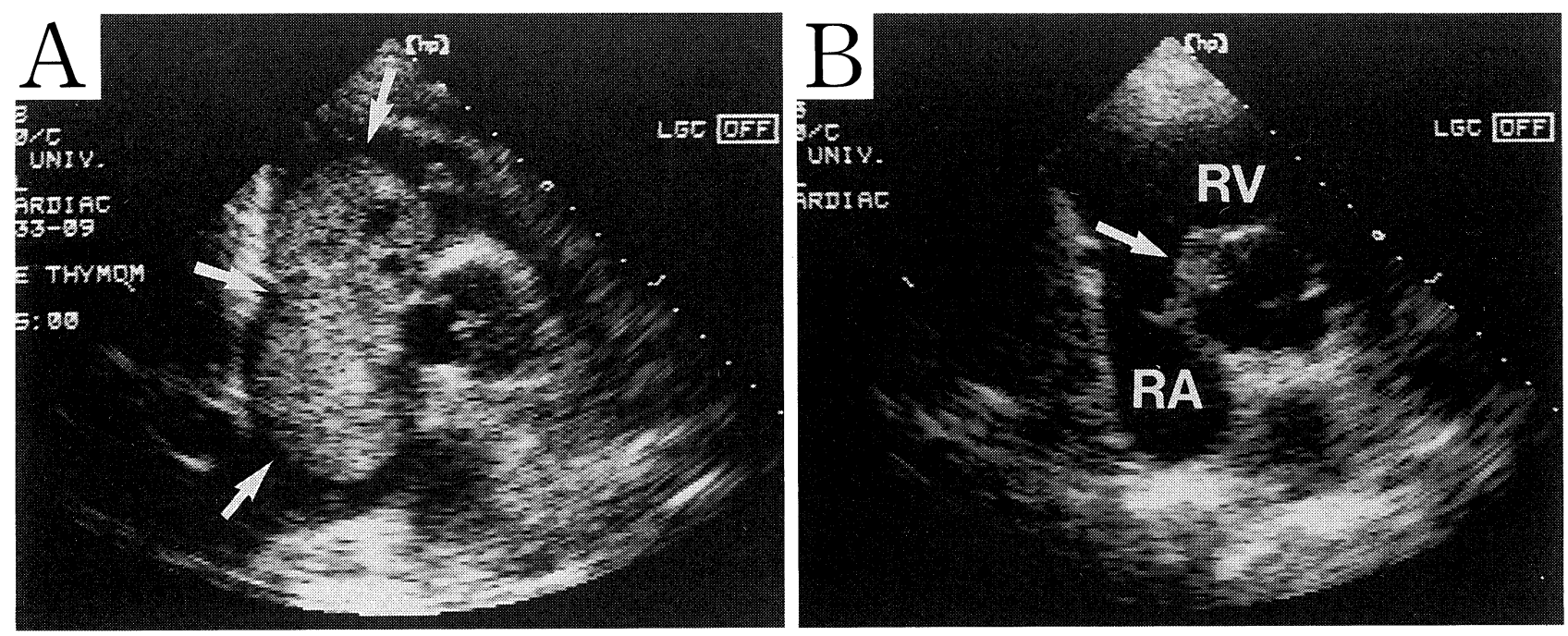

Figure 3. Preoperative (A) two-dimensional echocardiography demonstrating the intracardiac mass (white arrows) in the right atrium and right ventricle. Postoperative $(B)$ echocardiography showing the right atrium and right ventricle almost tumor-free. The remainder of the tumor is seen at the base of the atrial septum (white arrow).

moma. Postoperatively, the patient received adjuvant mediastinal irradiation (40Gy). A contrast enhanced CT scan of the thorax revealed a reduction of remaining tumors. Adjuvant chemotherapy was not done due to patient's refusal. Eighteen months after surgery, he remains free of symptoms.

\section{Discussion}

Cardiac involvement by metastatic neoplasms is found in 6 to $20 \%$ of autopsied patients who had malignant neoplasms (12). The most common primary malignancies encountered, in order of decreasing frequency, are lung, nonsolid primaries (lymphoma and leukemia), breast, stomach, skin, liver and colon (13). The epicardium is the region most often involved $(75.5 \%)$, followed by the myocardium (38.2\%) and endocardium (15.5\%) (14). Tumor extension into the vena cave and/or right atrium is sometimes observed in patients with renal cell carcinoma and hepatocellular carcinoma $(15,16)$. Superior vena cava obstruction and intracardiac extension of invasive thymoma have rarely been reported.

Gunn et al (8) reported a case of a malignant thymoma presenting with a retrosternal mass which was treated with surgery and radiotherapy. It recurred 5 years later as an intracardiac mass causing tricuspid valve obstruction. Although reoperation was performed in that case, it was impossible to remove the obstructing mass because of scarring caused by the previous procedure and radiotherapy. Thus, complete resection of the intracardiac mass seemed to be difficult in almost all previously reported cases. In our case also, surgical resection was performed twice but complete resection was difficult each time. However, circulatory improvement was achieved after the operation and our patient remains free of symptoms. The excision of intracardiac mass causing tricuspid valve ob- struction helped our patient barely escape sudden cardiac death. Although the follow-up after re-operation has been short, he remains free of symptoms. For this reason, we assume that the re-operation has been successful. We believe that, as recommended in the literature $(17,18)$, adjuvant chemotherapy and/ or radiotherapy would have improved his outcome. Early recognition of intracardiac involvement results in easier surgical treatment, therefore the present case prompts us to reflect that surgical excision should be done earlier rather than when symptoms are discovered due to blood flow occlusion. As was the case with our patient, intracavitary cardiac growth may be masked by the symptoms of superior or inferior vena cava syndromes (19). Therefore, serial examination by echocardiography is needed for patients with invasive thymoma but without cardiac symptoms or heart murmur.

Our case presented with recurrence of intracardiac mass without any change in the residual mass in the anterior mediastium, which constitutes an interesting mode of extension. This relatively slow growth may have been the result of its low grade malignancy. Hanfling (19) suggested that in cardiac involvement, especially tumor thrombosis, tumor cells might infiltrate the lumen of great veins by the initial accumulation of fibrin upon them. The fibrin serves as a framework for continued cancer growth, and the tumor thrombosis may then extend along the vein to the heart chambers, hindering blood flow.

In summary, we presented a rare case of invasive thymoma which re-extended into the intracardiac space three years postoperatively. Although cardiac involvement of an invasive thymoma is uncommon, intracavitary cardiac growth should be taken into consideration when treating patients with malignancies (20). 


\section{HAYASHI et al}

\section{References}

1) Rosai J, Levine GD. Tumors of the thymus. In: Harlan I, Ferminger MD, editors. Atlas of tumor pathology, Series 2, fascicle 13. Washington, D.C.: Armed forces Institute of Pathology. 1976: 1-221.

2) Lewis JE, Wick MR, Scheithauer BW, Bernatz PE, Taylor WF. Thymoma. A clinicopathologic review. Cancer 60: 2727-2743, 1987.

3) Filippone G, Savona I, Tomasello V, Guzzetta P, Zarcone N, Agate V. Radical excision of invasive thymoma with intracaval and intracardial extension. A successful case report. J Cardiovasc Surg 38: 547-549, 1997.

4) Okereke OUJ, Nzewi OC, Chikwendu VC, Odigwe E, Onuigbo WB. Radical excision of invasive thymoma with intracardiac extension. $\mathbf{J}$ Cardiovasc Surg 35: 355-358, 1994.

5) Futami $S$, Yamasaki $T$, Minami R, et al. Intracaval and intracardiac extension of malignant thymoma. Intern Med 32: 257-260, 1993.

6) Yokoi K, Miyazawa N, Mori K, et al. Invasive thymoma with intracaval growth into the right atrium. Ann Thorac Surg 53: 507-509, 1992.

7) Missault L, Duprez D, De Buyzere M, Cambier B, Adang L, Clement D. Right atrial invasive thymoma with protrusion through the tricuspid valve. Eur Heart J 13: 1726-1727, 1992.

8) Gunn J, Walker DR, Boyle RM. Malignant thymoma causing tricuspid valve obstruction. Eur Heart J 11: 854-856, 1990

9) Airan B, Sharma R, Iyer KS, et al. Malignant thymoma presenting as intracardiac tumor and superior vena caval obstruction. Ann Thorac Surg 50: 989-991, 1990.

10) Shimosato Y, Mukai K. Tumors of the mediastinum. Atlas of tumor pa- thology, Series 3, fascicle 21. Washington, D.C.: Armed forces Institute of Pathology. 1995: 40-120.

11) Kodama $T$, Watanabe $S$, Sato $Y$, Shimosato $Y$, Miyazawa N. An immunohistochemical study of thymic epithelial tumors. I. Epithelial component. Am J Surg Pathol 10: 26-33, 1986.

12) Ewald FW, Scherff AH. A 60-year-old man with a malignant tumor of the upper airway and unexplained respiratory failure. Chest 111: 239-241, 1997.

13) Abraham KP, Reddy V, Gattuso P. Neoplasms metastatic to the heart. Review of 3314 consecutive autopsies. Am J Cardiovasc Pathol 3: 195198, 1990.

14) Klatt EC, Heitz DR. Cardiac metastases. Cancer 65: 1456-1459, 1990.

15) Klein FA, Smith MJV, Greenfield LJ. Extracorporeal circulation for renal cell carcinoma with supradiaphragmatic vena caval thrombi. J Urol 131: 880-883, 1984.

16) Kato $Y$, Tanaka N, Kobayashi K, Ikeda T, Hattori N, Nonomura A. Growth of hepatocellular carcinoma into the right atrium. Ann Intern Med 99: 472-474, 1983.

17) Goldel N, Boning L, Fredrik A, Holzel D, Hartenstein R, Wilmanns W. Chemotherapy of invasive thymoma. A retrospective study of 22 cases. Cancer 63: 1493-1500, 1989.

18) Venuta F, Rendina EA, Pescarmona EO, et al. Multimodality treatment of thymoma. A prospective study. Ann Thorac Surg 64: 1585-1592, 1997.

19) Hanfling SM. Metastatic cancer to the heart. Review of the literature and report of 127 cases. Circulation 22: 474-483, 1960.

20) Yoshitomi $Y$, Kojima S, Sugi T, et al. Echocardiography of a right atrial mass in hepatocellular carcinoma. Heart Vessels 13: 45-48, 1998. 